\title{
Association of hepatitis B virus infection status with outcomes of non-small cell lung cancer patients undergoing anti-PD-1/PD-L1 therapy
}

\author{
Xuanye Zhang ${ }^{1,2,3 \#}$, Dan Tian ${ }^{4 \#}$, Yue Chen ${ }^{5 \#}$, Chen Chen ${ }^{1,2,6}$, Li-Na He ${ }^{1,2,3}$, Yixin Zhou ${ }^{1,2,7}$, Haifeng Li $^{1,2,3}$, \\ Zuan Lin ${ }^{1,2,8}$, Tao Chen ${ }^{1,2,9}$, Yuhong Wang ${ }^{1,2,10}$, Alessandro Russo ${ }^{11}$, Ernest Nadal ${ }^{12}$, Francesco Passiglia ${ }^{13}$, \\ Ross Andrew Soo ${ }^{14}$, Satoshi Watanabe ${ }^{15}$, Teresa Moran ${ }^{16}$, In-Jae Oh ${ }^{17}$, Sha Fu ${ }^{18}$, Shaodong Hong ${ }^{1,2,3}$, \\ Li Zhang ${ }^{1,2,3}$
}

${ }^{1}$ State Key Laboratory of Oncology in South China, Guangzhou, China; ${ }^{2}$ Collaborative Innovation Center for Cancer Medicine, Guangzhou, China; ${ }^{3}$ Department of Medical Oncology, Sun Yat-sen University Cancer Center, Guangzhou, China; ${ }^{4}$ Department of Thoracic Surgery, Guangdong Provincial People's Hospital, Guangdong Academy of Medical Sciences, Guangzhou, China; ${ }^{5}$ Departments of Dermatology, The Eighth Affiliated Hospital of Sun Yat-sen University, Shenzhen, China; ' Department of Radiation Oncology, Sun Yat-sen University Cancer Center, Guangzhou, China; ${ }^{7}$ Department of VIP Region, Sun Yat-sen University Cancer Center, Guangzhou, China; ${ }^{8}$ Department of Clinical Research, Sun Yat-sen University Cancer Center, Guangzhou, China; ${ }^{9}$ Department of Nuclear Medicine, Sun Yat-sen University Cancer Center, Guangzhou, China; ${ }^{10}$ Department of Endoscopy, Sun Yat-sen University Cancer Center, Guangzhou, China; ${ }^{11}$ Medical Oncology Unit, A.O. Papardo, Messina, Italy; ${ }^{12}$ Department of Medical Oncology, Catalan Institute of Oncology, IDIBELL, LHospitalet (Barcelona), Spain; ${ }^{13}$ Department of Oncology, University of Turin, S. Luigi Gonzaga Hospital, Orbassano, Italy; ${ }^{14}$ Department of Haematology-Oncology, National University Cancer Institute Singapore, National University Health System, Singapore, Singapore; ${ }^{15}$ Department of Respiratory Medicine and Infectious Diseases, Niigata University Graduate School of Medical and Dental Sciences, Niigata, Japan; ${ }^{16}$ Medical Oncology Department, Catalan Institute of Oncology, Hospital Universitari Germans Trias i Pujol, Badalona Applied Research Group in Oncology. Department of Medicine. Universitat Autònoma de Barcelona (UAB), Campus Can Ruti, Badalona, Spain; ${ }^{17}$ Department of Internal Medicine, Chonnam National University Medical School and Hwasun Hospital, Jeonnam, Korea; ${ }^{18}$ Guangdong Provincial Key Laboratory of Malignant Tumor Epigenetics and Gene Regulation, Pathology Department, Sun Yat-sen Memorial Hospital, Sun Yat-sen University, Guangzhou, China

Contributions: (I) Conception and design: S Hong, L Zhang; (II) Administrative support: S Hong, L Zhang; (III) Provision of study materials or patients: S Hong, X Zhang; (IV) Collection and assembly of data: X Zhang, Y Chen; (V) Data analysis and interpretation: X Zhang, D Tian, Y Chen; (VI) Manuscript writing: All authors; (VII) Final approval of manuscript: All authors.

\#These authors contributed equally to this work.

Correspondence to: Shaodong Hong; Li Zhang. 651 Dongfeng Road East, Guangzhou 510060, China. Email: hongshd@sysucc.org.cn; zhangli6@mail.sysu.edu.cn; Sha Fu. 33 Yingfeng Road, Guangzhou 510288, China. Email: luweizhijia@126.com.

Background: The aim of this study was to evaluate the safety and survival outcomes of anti-programmed cell death (PD)-1/programmed cell death-ligand 1 (PD-L1) monotherapy in patients with advanced nonsmall cell lung cancer (NSCLC) and different hepatitis B virus (HBV) infection status.

Methods: Patients with advanced NSCLC and both chronic and/or resolved HBV infection who were treated with anti-PD-(L)1 monotherapy were retrospectively enrolled. The primary endpoint was the safety of PD-1/PD-L1 monotherapy, while the secondary endpoints included the survival outcomes.

Results: Of the 62 eligible patients, 10 (16.1\%) were hepatitis B surface antigen (HBsAg) positive [chronic hepatitis $\mathrm{B}(\mathrm{CHB})$ infection] and $52(83.9 \%)$ were HBsAg negative and HBcAb positive [resolved hepatitis B (RHB) infection]; 42 (67.7\%) patients had at least 1 treatment-related adverse event (AE), with 4 patients (6.5\%) developing grade 3 AEs and 6 (9.7\%) developing hepatic AEs. One CHB patient experienced HBV reactivation during anti-PD-1 immunotherapy due to the interruption of antiviral prophylaxis. The objective response rate and durable clinical benefit (DCB) rate were $17.7 \%$ and $29.0 \%$, respectively. Median overall survival (OS) and progression-free survival (PFS) were 23.6 months [95\% confidence interval (CI): $14.4-$ 32.8] and 2.1 months (95\% CI: 1.2-3.0), respectively. The DCB rate was significantly higher in the CHB group than in the RHB group (60\% vs. $23.1 \%$; $\mathrm{P}=0.048)$. Patients with $\mathrm{CHB}$ experienced a longer PFS (8.3 
vs. 2.0 months; $\mathrm{P}=0.103$ ) and $\mathrm{OS}(35.0$ vs. 18.2 months, $\mathrm{P}=0.119)$ than did $\mathrm{RHB}$ patients.

Conclusions: Anti-PD-(L)1 monotherapy was safe and effective in patients with NSCLC and HBV infection. This population should not be excluded from receiving immunotherapy in routine clinical practice or within clinical trials if HBV biomarkers are monitored and antiviral prophylaxis is properly used.

Keywords: Non-small cell lung cancer (NSCLC); programmed cell death 1 (PD-1); programmed cell deathligand 1 (PD-L1); immunotherapy; hepatitis B virus infection (HBV infection)

Submitted Mar 07, 2021. Accepted for publication Jun 24, 2021.

doi: $10.21037 /$ tlcr-21-455

View this article at: https://dx.doi.org/10.21037/tlcr-21-455

\section{Introduction}

Lung cancer remains the leading cause of cancerassociated mortality (1). Recently, anti-programmed cell death 1 (PD-1) and anti-programmed cell death-ligand 1 (PD-L1) blockade have revolutionized the treatment of various malignancies, including non-small cell lung cancer (NSCLC). Several PD-(L)1 pathway inhibitors have been approved for second-line treatment of advanced NSCLC patients after progression on platinum-based therapy (2-5). For patients with high PD-L1 expression, pembrolizumab, atezolizumab, and cemiplimab have replaced platinum doublet chemotherapy as the first-line treatment with a better toxicity profile while retaining survival benefit (6-8). However, patients with NSCLC and some concurrent infections, including human immunodeficiency virus (HIV) infection and hepatitis $\mathrm{B} / \mathrm{C}$ infection, were excluded from most clinical trials of immunotherapy. Recently, some retrospective studies or prostective clinical trials have demonstrated that the efficacy and limited toxicity of PD(L)1 inhibitors in patients with HIV infection and advanced NSCLC is similar to non-infected patients (9-13). However, data from the literature have been limited to case reports or small case series that have described the utilization of PD-1 pathway inhibitor (alone or in combination with ipilimumab or chemotherapy) for advanced NSCLC patients with chronic or previous HBV infection (14-20). Therefore, there is a paucity of information on the safety and efficacy of anti-PD-(L) 1 monotherapy in this population.

More than 350 million people are chronically infected with $\mathrm{HBV}$ worldwide $(21,22)$. Apart from being involved in cancer immune evasion (23), the PD-1/PD-L1 axis also plays a role in chronic hepatitis virus infection. Previous studies have shown that PD-1 upregulation is associated with HBV-specific T cell dysfunction $(24,25)$, while tumoral PD-L1 expression in hepatocellular carcinoma has been associated with circulating HBV load (26). For NSCLC, PD-L1 expression has also been reported to be significantly higher in patients with chronic HBV infection than in those without HBV infection (27). Safety is another critical concern. In our previous study, HBV reactivation occurred in a subset of patients with cancer and positive hepatitis B surface antigen (HBsAg) during or after anti-PD-(L)1 therapy (6 out of 114 patients) (28).

Considering the scale of HBV infection in the global population, the under representation of $\mathrm{HBV}$-infected patients in clinical trials, the potential association between PD-L1 expression and HBV infection, and the related safety issues, we conducted this retrospective study to explore whether PD-1 pathway inhibitors are safe and effective in patients with advanced NSCLC and different HBV infection status. We present the following article in accordance with the STROBE reporting checklist (available at https://dx.doi.org/10.21037/tlcr-21-455).

\section{Methods}

Between January 2015 and September 2018, a total of 174 patients with advanced NSCLC who received anti-PD(L)1 monotherapy in Sun Yat-sen University Cancer Center were screened. The inclusion criteria were as follows: (I) pathologically diagnosed with NSCLC; (II) stage IIIB or IV NSCLC or recurrent NSCLC after radical treatment; (III) received at least 1 cycle of anti-PD-(L)1 monotherapy; (IV) serum HBsAg positive or HBsAg negative and hepatitis B core antibody ( $\mathrm{HBcAb})$ positive; and $(\mathrm{V})$ with complete clinical and survival data. Patients were excluded if they had other positive viral markers including immunoglobin M (IgM) antibody to hepatitis A virus, antibody to hepatitis C virus (HCV), IgG antibody to hepatitis D virus, $\operatorname{IgM}$ antibody to hepatitis E virus, or antibody to human 
immunodeficiency virus.

Clinicopathologic data and treatment outcomes were retrospectively collected. All patients were followed up by outpatient reviews or by telephone conversations, with the last follow-up date being June 1, 2019. Response to treatment was assessed with Response Evaluation Criteria in Solid Tumors (RECIST) criteria version 1.1 (29) by senior radiologists blinded to patients' data, and consisted of complete response (CR), partial response (PR), stable disease (SD), and progressive disease (PD). Follow-up computed tomography (CT) scans were performed according to the physicians' discretion without predetermined intervals. Objective response was defined as complete or partial response, disease control was defined as $\mathrm{CR}+\mathrm{PR}+\mathrm{SD}$, and durable clinical benefit (DCB) was defined as survival without disease progression at 24 weeks. Progression-free survival (PFS) was defined as the time from the initiation of anti-PD-(L)1 monotherapy to the date of disease progression or death from any causes. Overall survival (OS) was defined as the time from the beginning of anti-PD-(L)1 monotherapy to the date of death from any causes. Treatment-related adverse events (AEs) were assessed according to the Common Terminology Criteria for Adverse Events (CTCEA) version 4.0. HBV reactivation was defined according to the American Association for the Study of Liver Diseases (AASLD) 2018 hepatitis B guidance (30) and included the following: (I) a $\geq 2 \log$ (100-fold) increase in HBV DNA compared to the baseline level, (II) HBV DNA $\geq 3 \log (1,000) \mathrm{IU} / \mathrm{mL}$ in a patient with a previously undetectable level, or (III) HBV DNA $\geq 4 \log (10,000) \mathrm{IU} / \mathrm{mL}$ if the baseline level was not available.

The study was conducted in accordance with the Declaration of Helsinki (as revised in 2013). This study was approved by the Sun Yat-sen University Cancer Center Institutional Review Board (No. B2020-402-01), and the need for informed consent (written or verbal) was waived.

\section{Statistical analysis}

The primary endpoint of this study was the safety and tolerability of PD-(L)1 inhibitors in $\mathrm{HBV}$-infected patients with advanced NSCLC. The secondary endpoint was the antitumor activity of PD-(L)1 inhibitors in this population, including overall response rate (ORR), disease control rate (DCR), DCB rate, PFS and OS. Baseline characteristics were tabulated and summarized. All treatment-related AEs that could be possibly attributed to anti-PD-(L)1 immunotherapy were tabulated. The Pearson $\chi^{2}$ or Fisher exact test was used to investigate the relationships between categorical variables. Survival analyses were performed using the Kaplan-Meier method with log-rank test. A 2 -tailed $\mathrm{P}$ value of $\leq 0.05$ was used to determine statistical significance. All statistical analyses were performed using SPSS version 22.0 (IBM, Armonk, NY, USA).

\section{Results}

\section{Patients}

A total of 62 eligible patients were included in the analysis. Baseline characteristics of the patients are summarized in Table 1. All of the 62 patients were Asian. The median age was 55 (range, 33-77) years, and 45 (72.6\%) patients were male. In terms of the histology subtype, 38 (61.3\%) patients had adenocarcinoma, 20 (32.3\%) patients had squamous cell carcinoma, and 4 (6.4\%) patients had lymphoepitheliomalike carcinoma; 25 patients (40.3\%) were current or former smokers, 60 (96.8\%) had an Eastern Cooperative Oncology Group (ECOG) performance status 0 or $1 ; 10$ patients (16.1\%) were HBsAg positive [chronic hepatitis B (CHB) infection], while 52 patients (83.9\%) were HBsAg negative and $\mathrm{HBcAb}$ positive [resolved hepatitis $\mathrm{B}$ (RHB) infection]. Of the 10 patients with CHB, 9 (90.0\%) patients had a baseline HBV DNA quantification which was undetectable, with 8 of these patients being on antiviral prophylaxis prior to and during PD-1 axis inhibition (6 patients received entecavir and 2 received lamivudine). Of the 52 patients with RHB, only 6 (11.5\%) had baseline HBV DNA data and all were undetectable, with none of these patients receiving antiviral prophylaxis. Before anti-PD-(L)1 monotherapy, 57 (91.9\%) had received previous systemic therapy, and the median number of previous lines of therapy was 1 (range, $0-7)$. Only 8 patients had a quantifiable tumor PD-L1 expression with the $22 \mathrm{C} 3$ assay, and 4 of them had a PD-L1 expression $\geq 1 \%$.

\section{Safety outcomes}

Treatment-related AEs are listed in Table 2. In the overall population, $42(67.7 \%)$ of 62 patients had at least 1 treatment-related AE. The most common AEs of any grade were reactive capillary hemangiomas $(n=15,24.2 \%$, related to camrelizumab only), rash $(\mathrm{n}=14,22.6 \%)$, fatigue $(\mathrm{n}=13$, $21.0 \%)$, fever $(\mathrm{n}=8,12.9 \%)$, hypothyroidism $(\mathrm{n}=7,11.3 \%)$, alanine aminotransferase (ALT) increase $(n=6,9.7 \%)$, and 
Table 1 Baseline characteristics of the included patients

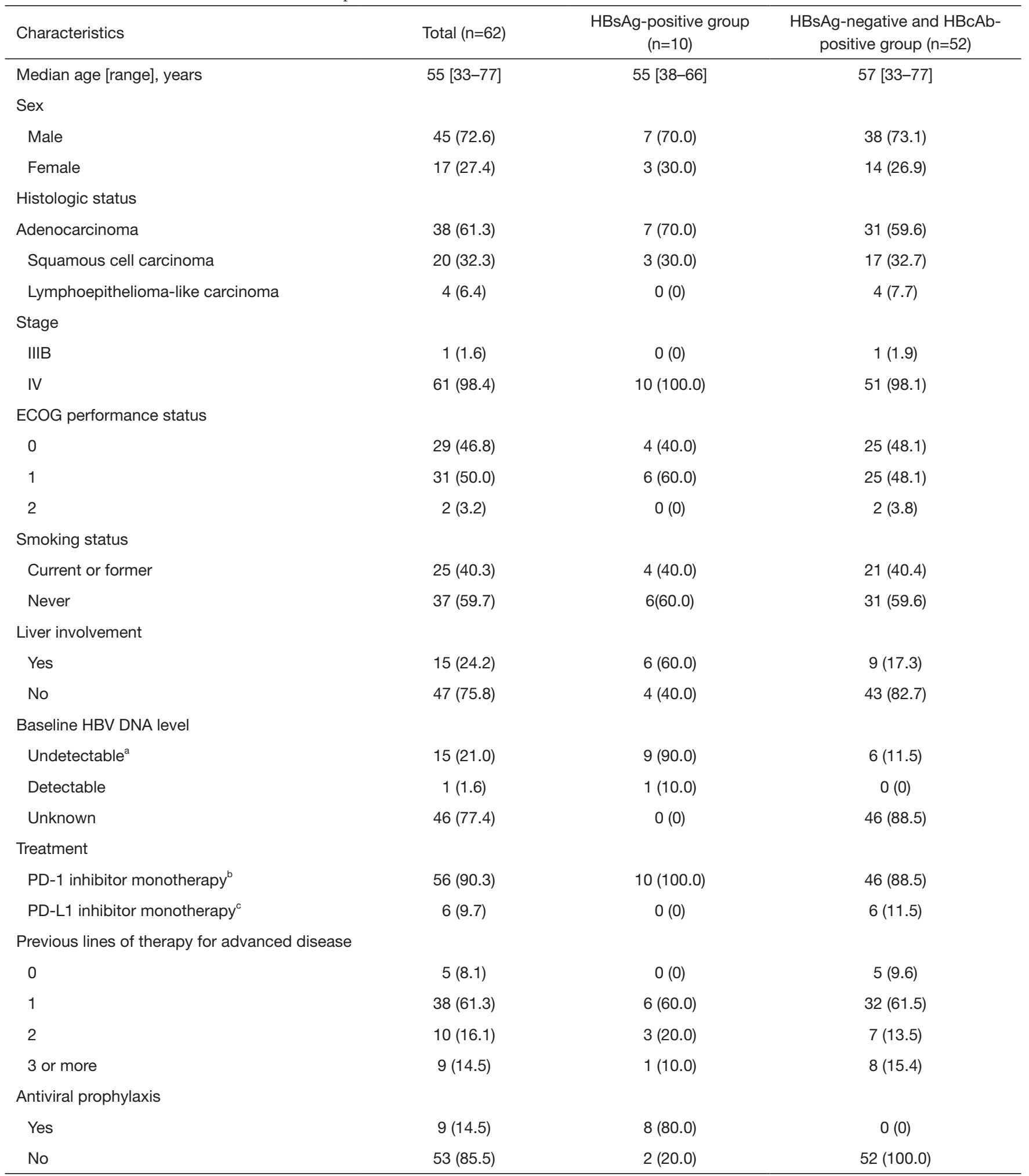

a , HBV DNA <10 IU/mL; ' , included pembrolizumab, nivolumab, and camrelizumab; ${ }^{\text {, }}$, atezolizumab. HBsAg, hepatitis B surface antigen; HBcAb, hepatitis B core antibody; ECOG, Eastern Cooperative Oncology Group; HBV, hepatitis B virus; PD-1, programmed cell death protein-1; PD-L1, programmed cell death-ligand 1. 
Table 2 Treatment-related adverse events at least possibly related to PD-1/PD-L1 immunotherapy

\begin{tabular}{|c|c|c|c|c|}
\hline Event & Grade 1, n (\%) & Grade 2, n (\%) & Grade 3, n (\%) & Total, n (\%) \\
\hline Reactive capillary hemangiomas & $9(14.5)$ & $6(9.7)$ & 0 & $15(24.2)$ \\
\hline Rash & $8(12.9)$ & $6(9.7)$ & 0 & $14(22.6)$ \\
\hline Fatigue & $9(14.5)$ & $3(4.8)$ & $1(1.6)$ & $13(21.0)$ \\
\hline Hypothyroidism & $5(8.1)$ & $2(3.2)$ & 0 & $7(11.3)$ \\
\hline ALT increase & $4(6.5)$ & $1(1.6)$ & $1(1.6)$ & $6(9.7)$ \\
\hline AST increase & $4(6.5)$ & $1(1.6)$ & $1(1.6)$ & $6(9.7)$ \\
\hline Pruritus & $4(6.5)$ & 0 & 0 & $4(6.5)$ \\
\hline Dizziness & $3(4.8)$ & 0 & 0 & $3(4.8)$ \\
\hline Anorexia & $3(4.8)$ & 0 & 0 & $3(4.8)$ \\
\hline Stomatitis & $2(3.2)$ & 0 & 0 & $2(3.2)$ \\
\hline Hyperthyroidism & $2(3.2)$ & 0 & 0 & $2(3.2)$ \\
\hline Peripheral neuropathy & 0 & 0 & $1(1.6)$ & $1(1.6)$ \\
\hline Myasthenia Gravis & 0 & 0 & $1(1.6)$ & $1(1.6)$ \\
\hline Anemia & $1(1.6)$ & 0 & 0 & $1(1.6)$ \\
\hline Nausea & $1(1.6)$ & 0 & 0 & $1(1.6)$ \\
\hline
\end{tabular}

a, worst per patient. ALT, alanine aminotransferase; AST, aspartate aminotransferase; PD-1, programmed cell death protein-1; PD-L1, programmed cell death-ligand 1.

aspartate aminotransferase (AST) increase $(n=6,9.7 \%)$. Although most AEs were grade 1 or $2(\mathrm{n}=38,61.3 \%), 4$ (6.5\%) patients developed grade 3 AEs, including 1 case of peripheral neuropathy, 1 case of myasthenia gravis, 1 case of ALT and AST increase, and 1 case of fatigue. All grades of hepatic AEs occurred in 6 (9.7\%; grade $1-2$ in 5 patients and grade 3 in 1 patient) patients, all of whom experienced laboratory abnormalities without any symptoms. Immunotherapy was permanently discontinued in $3(4.8 \%)$ patients due to treatment-related AEs, and no treatment-related death occurred during the study period. No significant differences were found in any grade of AEs between patients with $\mathrm{CHB}$ and RHB; however, the incidence of hepatic AEs was significantly higher in the CHB group than in the RHB group $(50 \%$ vs. $1.9 \%$; $\mathrm{P}<0.001$; Table 3).

\section{Immunotherapy-related $\mathrm{HBV}$ reactivation}

Among the 10 patients with $\mathrm{CHB}$, serum HBV DNA was monitored every 1 to 3 months according to the decision of the treating physician. One (10\%) patient experienced 
Table 3 The comparison of safety and efficacy of PD-1/PD-L1 immunotherapy between the HBsAg-positive group and the HBsAg-negative but $\mathrm{HBcAb}$-positive group

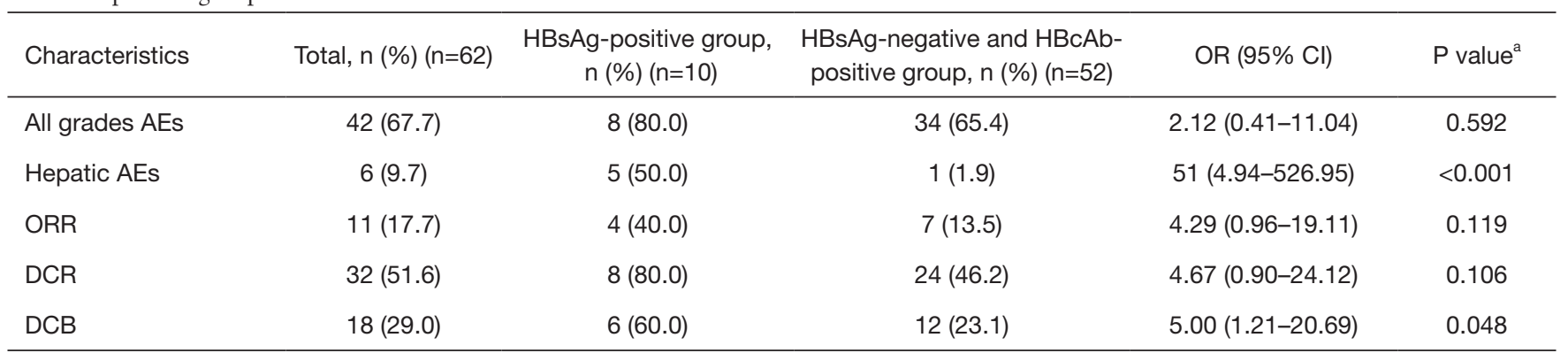

a , determined using the $\chi^{2}$ test. AE, adverse event; PD-1, programmed cell death protein-1; PD-L1, programmed cell death-ligand 1; HBsAg, hepatitis B surface antigen; HBcAb, hepatitis B core antibody; OR, odds ratio; Cl, confidence Interval; ORR, objective response rate; $D C R$, disease control rate; $D C B$, durable clinical benefit.

HBV reactivation during the study period. For the other 9 patients, their HBV DNA level kept undetectable during the whole study period. Of the 52 patients with RHB, no one developed HBsAg reverse seroconversion.

The patient was a 41-year-old woman diagnosed with stage IV NSCLC in July 2014. She began camrelizumab monotherapy in December 2016 following progression with carboplatin and pemetrexed. She had previously been diagnosed with a chronic HBV infection with an unknown HBV DNA level. Her baseline HBV DNA level in the month before treatment with camrelizumab was 40.6 IU/mL. Camrelizumab administration was commenced in December 2016 along with entecavir antiviral therapy. In February 2017, after 2 months of camrelizumab and entecavir treatment, a repeat HBV viral load showed undetectable DNA. The patient continued entecavir therapy and maintained an undetectable HBV DNA level until the 25th cycle of camrelizumab treatment in October 2017, at which time she stopped entecavir against the physician's recommendation. After 36 cycles of camrelizumab, a rise in ALT to $530.3 \mathrm{U} / \mathrm{L}$ and AST to $312.0 \mathrm{U} / \mathrm{L}$ was noticed on July 2, 2018. Total bilirubin and alkaline phosphatase remained within normal ranges. The HBV DNA level increased to $2.18 \times 10^{3} \mathrm{IU} / \mathrm{mL}$, confirming the occurrence of HBV reactivation. Consequently, camrelizumab was discontinued and entecavir was reintroduced. ALT and AST started to trend downward immediately after entecavir administration, reaching grade 1 - in a 2 -week period. HBV DNA returned to an undetectable level after 5 weeks. The patient resumed camrelizumab after 4 weeks of interruption without any further significant AEs occurring.

\section{Efficacy}

In all, 56 of 62 patients (90.3\%) received PD-1 inhibitor (pembrolizumab, nivolumab, and camrelizumab) and 6 patients $(9.7 \%)$ received PD-L1 inhibitor (atezolizumab). The median duration of PD-(L)1 inhibitor treatment was 10 weeks (range, $1-157$ weeks). With a median follow-up time of 28.4 months (range, 0.6-53.2 months), the median OS of the 62 patients was 23.6 months (95\% CI: $14.4-32.8$; Figure 1). The OS rate at 1 year and 2 years was $73.0 \%$ and $49.7 \%$, respectively. Eleven patients achieved PR, and no patients had complete response, yielding an ORR of $17.7 \%$. SD was observed in $21(33.9 \%)$ patients, and thus disease control was observed in $33(53.2 \%)$ patients. The DCB rate was $29.0 \%$. Among the 11 patients with a confirmed objective response, the median time to response was 9 weeks (range, 4-47 weeks), and the median duration of response was 16.2 months (95\% CI: 10.3-22.1). The median PFS was 2.1 months (95\% CI: 1.2-3.0; Figure 1).

The DCB rate was significantly higher in patients with CHB than in those with RHB (60\% vs. $23.1 \%$; $\mathrm{P}=0.048)$. The CHB group received more clinical benefit from antiPD-(L)1 monotherapy as compared to the RHB group, in terms of ORR ( $40 \%$ vs. $13.5 \%$; $\mathrm{P}=0.119)$, DCR $(80 \%$ vs. 46.2\%; $\mathrm{P}=0.106)$, median PFS (8.3 vs. 2.0 months; $\mathrm{P}=0.103)$, and $\mathrm{OS}$ (35.0 vs. 18.2 months; $\mathrm{P}=0.119)$. These results are shown in Table 3 and Figure 1.

\section{Discussion}

PD-1 pathway inhibitors have become a cornerstone in the treatment of patients with nononcogene addicted advanced 
A

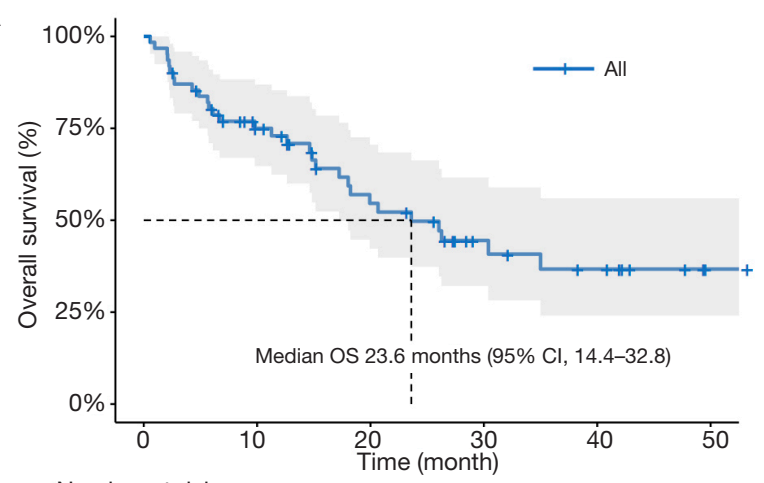

Number at risk

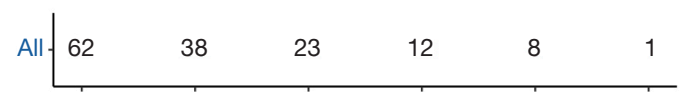

C
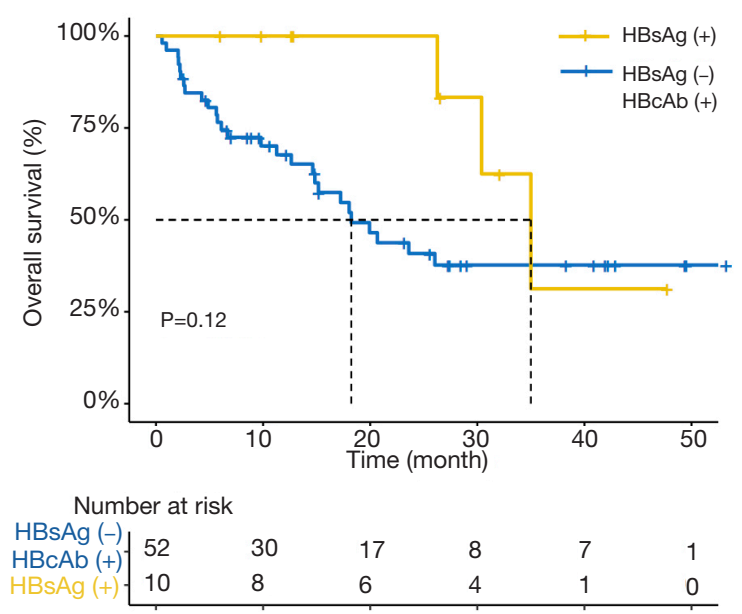

B

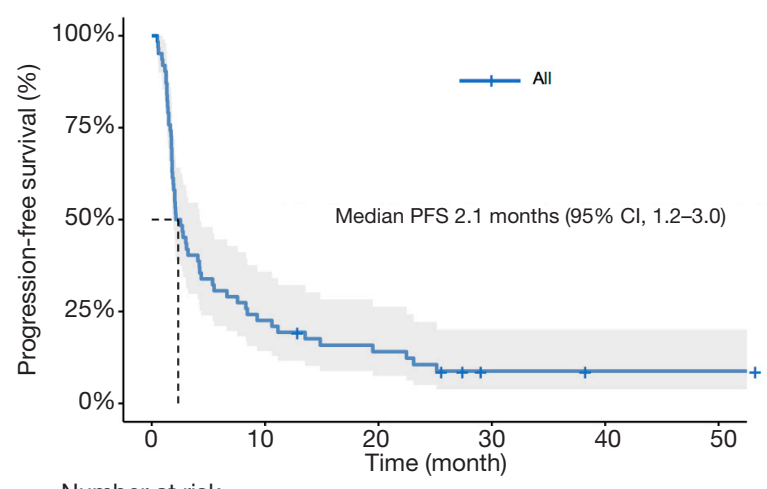

Number at risk

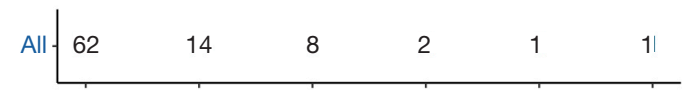

$D$

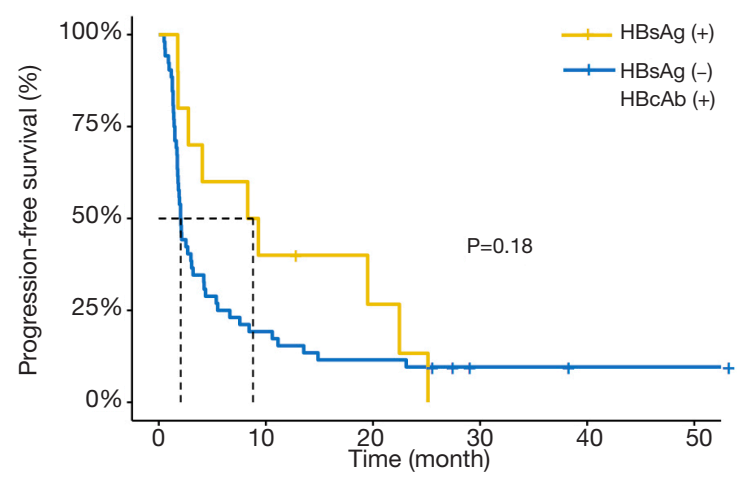

Number at risk

\begin{tabular}{|c|c|c|c|c|c|c|}
\hline $\begin{array}{l}\text { HBsAg (-) } \\
\text { HBcAb }(+)\end{array}$ & 52 & 10 & 6 & 2 & 1 & 1 \\
\hline $\mathrm{HBsAg}(+)$ & 10 & 4 & 2 & 0 & 0 & 0 \\
\hline
\end{tabular}

Figure 1 Kaplan-Meier analysis of overall survival (OS) and progression-free survival (PFS). (A) OS of 62 enrolled patients. (B) PFS of 62 enrolled patients. (C) OS according to hepatitis B surface antigen (HBsAg) status. (D) PFS according to HBsAg status.

NSCLC. However, patients with HBV infection have systematically been excluded from immunotherapy clinical trials, limiting our understanding concerning the safety and efficacy of immune checkpoints inhibitors (ICIs) among these patients. Several case reports have demonstrated that PD-(L) 1 inhibitors can be safe and effective in NSCLC patients with either chronic HBV or HCV infection (14-20). Most of the relevant studies have enrolled a limited number of patients, impeding in-depth characterization of the safety and efficacy of immunotherapy. To the best of our knowledge, this is the largest study to detail the safety and efficacy of PD-(L)1 inhibitors in patients with advanced NSCLC and different HBV infection status. Our results showed that anti-PD-(L)1 monotherapy yielded similar efficacy outcomes in NSCLC patients with HBV infection as those observed in patients without HBV $(2-4,31)$. The incidence of all-grade hepatic AEs in this cohort (9.7\%) seems to be slightly higher than that reported in previous clinical trials (4-6,32), while other AE profiles were similar. $\mathrm{HBV}$ reactivation was identified in $1 \mathrm{CHB}$ patient during anti-PD-1 therapy after the antiviral agent was stopped by the patient.

In the CA209-003 study involving HBV-negative NSCLC patients who had received at least 1 prior systemic anticancer regimen, the response rate to nivolumab monotherapy was $17 \%$, with a median duration of response of 17.0 months $(33,34)$, a median OS of 9.9 months, and 1-, 2-, and 3-year OS rates of $42 \%, 24 \%$, and $18 \%$, 
respectively $(23,24)$. In our study, with $91.9 \%$ pretreated participants, the overall response rate was $17.7 \%$, the DCB rate was $29.0 \%$, the median duration of response was 16.2 months, the median OS was 23.6 months, and the 1and 2 -year OS rates were $73.0 \%$ and $49.7 \%$, respectively. Another retrospective case series enrolled 19 patients with NSCLC and a history of HBV or HCV infection and found that the ORR in 17 patients treated with immunotherapy alone was $35 \%$ and the median PFS was 4.5 months (18). These results suggest that the response rate and longterm outcome of PD-(L)1 inhibitors in HBV-infected NSCLC patients appear to be similar to those without $\mathrm{HBV}$ infection. Interestingly, the clinical benefit from ICI among patients varied depending on the HBV infection status. In this study, we found that patients with chronic HBV infection had a significantly higher DCB rate than those with a resolved $\mathrm{HBV}$ infection $(60 \%$ vs. $23.1 \%$; $\mathrm{P}=0.048)$. There also was a tendency toward improved ORR and PFS in patients with chronic HBV infection. In a retrospective study enrolling NSCLC patients with a history of tuberculosis (TB) and/or HBV who were treated with ICI (monotherapy or combination therapy), patients in the group with HBV or TB appeared to have better survival outcomes compared with the group without, in terms of PFS (5.7 vs. 3.1 months; $\mathrm{P}=0.021)$ and $\mathrm{OS}(15.6$ vs. 11.1 months, $\mathrm{P}=0.046$ ) (19). Of note, the group with TB or HBV included a higher proportion of patients receiving ICI in the first-line setting with PD-L1 expression $\geq 1 \%$. Considering the retrospective nature and small sample size of the current study, our findings should be interpreted with caution. In NSCLC patients, the tumor-expressed ligands PD-L1 and PD-L2 engage the PD-1 receptor expressed on activated $\mathrm{T}$ cells to down-regulate T-cell activation (23). Meanwhile, upregulation of PD-1 on viral specific T cells from $\mathrm{CHB}$ patients has been observed and proven to be associated with $\mathrm{T}$ cell exhaustion and persistent viral infection $(24,25)$. The finding of higher PD-L1 expression in tumor cells also indicates that there might be an HBV-mediated systemic immune response that could potentially affect the anti-/PD-(L)1 immunotherapy in NSCLC patients with CHB (27). PD-1 inhibitor administration might restore $\mathrm{T}$ cell function, and the cytotoxic $\mathrm{T}$ cells may induce antitumor activity more effectively in patients with CHB.

With anti-PD-(L) 1 monotherapy, the incidence of hepatic AEs in HBV-negative NSCLC patients is about 6\% or less in reported studies, with grade 3-4 events occurring in $1-2 \%$ of patients $(5,32,35)$. These hepatic AEs consist mainly of asymptomatic elevations in AST and ALT levels.
In our study, all grade and grade 3-4 hepatic AEs occurred in $9.7 \%$ and $1.6 \%$ of the patients, respectively. Recently, the nonrandomized, open-label, real-world, CheckMate870 trial (NCT03195491), examined the safety and efficacy of nivolumab as second-line therapy for Asian patients with NSCLC (36). One of the secondary endpoints was the safety of nivolumab in HBV-infected patients (a total of 17 patients required to have an HBV DNA level of lower than $500 \mathrm{IU} / \mathrm{mL}$ ). The most common grade 3-4 AEs included those observed in the liver $(2.3 \%)$, skin (1.6\%), lungs $(1.0 \%)$, and endocrine system $(0.8 \%)$. The ORR was similar between those with and without HBV infection (17.6\% vs. 15.4\%). Taken together, anti-PD-(L)1 monotherapy is generally well tolerated in patients with NSCLC and a concurrent HBV infection.

In addition to their antitumor activity, anti-PD-(L)1 antibodies' effect on the control of chronic viral infections is of immense interest. A clinical trial (NCT00703469) that assessed an anti-PD-1 antibody in patients with chronic $\mathrm{HCV}$ infection showed that some patients have persistent suppression of HCV replication. However, there are case reports or case series indicating that $\mathrm{HBV}$ reactivation is a potential complication of anti-PD-(L)1 therapy in HBVinfected patients with cancers $(16,17,19,28,37)$. In our previous pan-cancer study, we found that HBV reactivation occurs in $5.3 \%$ of the patients with positive HBsAg, and that the lack of prophylactic antiviral therapy was the most important risk factor [odds ratio (OR) 17.50] (28). In the current study, an incident of HBV reactivation was identified in an HBsAg-positive patient receiving camrelizumab. Furthermore, several isolated cases of anti-PD-1 immunotherapy-related HBV reactivation in patients with resolved HBV infection have been reported $(16,17,37)$. These findings suggest that both patients with chronic $\mathrm{HBV}$ and resolved $\mathrm{HBV}$ infection are at risk of virus reactivation while receiving $\mathrm{PD}-(\mathrm{L}) 1$ blockade, and that screening for $\mathrm{HBV}$ in all patients should be performed before treatment begins (38). It's worth noting that, though widely observed in clinical practice, the mechanism of $\mathrm{HBV}$ reactivation induced by anti- PD-1/PD-L1 therapy is still unclear. More basic research will be needed to reveal the underlying mechanisms. For the patient experienced $\mathrm{HBV}$ reaction in the current study, entecavir was given as prophylaxis at the commencement of camrelizumab, but was stopped disregarding the physician's advice after 25 cycles of immunotherapy. The HBV reactivation event occurred 33 weeks after the interruption of entecavir. This case supports the monitoring of HBV DNA level and 
maintenance of antiviral prophylaxis before and during anti-PD-(L)1 immunotherapy as a clinically important measure in CHB patients. A recent report indicates that concurrent antiviral prophylaxis might allow safe treatment even in patients with high HBV DNA loads (>500 IU/mL) (39). Our previous study also found 1 event of reactivation in a patient with $\mathrm{CHB}$ that occurred 6 weeks after immunotherapy was discontinued (28), implying that antiviral treatment should be continued even after the PD-(L)1 inhibitor administration has been completed. According to AASLD guidance (30), the administration of antiviral therapy should last for at least 6 months after completion of immunosuppressive agents or chemotherapy, but whether this recommendation is applicable for immunotherapy remains to be determined. For RHB patients, who are at lower risk of $\mathrm{HBV}$ reactivation than are CHB patients (19), treatment can begin on prophylaxis, or ALT, HBV DNA, and HBsAg can be carefully monitored with the intent for on-demand anti-HBV therapy (30).

Given our experience in this real-world study, anti-PD(L)1 immunotherapy appears to be safe and effective in HBV-infected patients with NSCLC. However, further research is warranted to address several unanswered questions regarding the application of ICIs in patients with NSCLC and HBV infection. An in-depth characterization of the tumor microenvironment, especially PD-L1 expression in NSCLC for patients with HBV, is currently lacking. Furthermore, the safety of ICIs in this population deserves further investigation with regards to serious immune-related AEs and particularly $\mathrm{HBV}$ reactivation. Although we had previously shown that a lack of prophylactic antiviral therapy is a significant risk factor of $\mathrm{HBV}$ reactivation, the underlying mechanisms remain unclear. Also, how HBV infection impacts the systemic immune response and the action of PD-(L) 1 inhibitors in cancer patients should be further studied. Finally, the response patterns of anti-/PD-(L)1 therapy and factors that might impact the likelihood of response are also unknown.

Some limitations to our study should be addressed. First, as a retrospective, single-center study, there might have been bias in the selection of patients. The results need to be further validated by future clinical studies with larger sample sizes. Second, only patients with $\mathrm{CHB}$ were regularly monitored for HBV DNA in this study, while patients with RHB were not routinely tested for HBV DNA before and during anti-PD-(L)1 immunotherapy. The lack of data concerning HBV DNA level could have resulted in an underdetection of the reactivation events in these patients. Given its clear value in monitoring viral reactivation in $\mathrm{HBV}$-infected patients, we advise incorporating baseline and on-treatment HBV DNA levels into routine clinical practice. Finally, the measurement of tumoral PD-L1 expression was not performed in most patients. Thus, we could not further evaluate the association between HBV infection and PD-L1 expression in NSCLC patients.

\section{Conclusions}

Our study indicates that anti-PD-(L)1 immunotherapy could be safe and effective in $\mathrm{HBV}$-infected patients. Patients with positive HBsAg (chronic HBV infection) were more likely to have durable benefit than those with resolved HBV infection. HBV reactivation may occur upon treatment with $\mathrm{PD}-(\mathrm{L}) 1$ inhibitors, and this should be monitored attentively throughout the therapeutic process. Our findings support the use of anti-PD-(L)1 immunotherapy in these patients, along with regular monitoring of HBV serological markers and properly administrated antiviral prophylaxis to prevent HBV reactivation. Future clinical trials of anti-PD(L)1 immunotherapy should not exclude patients with HBV infection. Further studies are needed to better characterize the response patterns of immunotherapy in this special population of $\mathrm{HBV}$-infected patients.

\section{Acknowledgments}

We sincerely appreciate all the patients and their families who were included in this retrospective study. The authors would also like to thank the AME Lung Cancer Collaborative Group for their academic support.

Funding: This study was funded by grants from the National Natural Science Funds of China (no. 81972898 and 81872499), the Natural Science Funds of Guangdong Province (no. 2019A1515011090), and the Outstanding Young Talents Program of Sun Yat-sen University Cancer Center (no. 16zxyc04). The funding sources had no role in the design and conduct of the study; collection, management, analysis, and interpretation of the data; preparation, review, or approval of the manuscript; or decision to submit the manuscript for publication.

\section{Footnote}

Reporting Checklist: The authors have completed the STROBE reporting checklist. Available at https://dx.doi. 
org/10.21037/tlcr-21-455

Data Sharing Statement: Available at https://dx.doi. org/10.21037/tlcr-21-455

Conflicts of Interest: All authors have completed the ICMJE uniform disclosure form (available at https://dx.doi. org/10.21037/tlcr-21-455). AR reports advisory board role for Astra Zeneca, MSD, and Novartis. RAS reports research grants from Astra-Zeneca, Boehringer Ingelheim, and advisory Board for Amgen, Astra-Zeneca, Bayer, BMS, Boehringer Ingelheim, Lilly, Merck, Novartis, Pfizer, Roche, Taiho, Takeda, Yuhan. EN received research funding from Roche, Pfizer, Merck-Serono, and Bristol Myers Squibb and received consultancy fees from Roche, BMS, MSD, Merck-Serono, Pfizer, Lilly, Amgen, BoehringerIngelheim, AstraZeneca, and Takeda. FP reports Consulting fees from MSD, Astrazeneca, Boehringer Ingheleim, Amgen and Pfizer. TM reports honorara for lecture and edcuational presentations as well as consultin fees from Astra Zeneca, Lilly, MSD, BMS. IJO received research funding from Roche and received consultancy fees from Roche, Ono, MSD, Pfizer, Boehringer-Ingelheim, AstraZeneca, Takeda. SW reports grant and personal fees from AstraZeneca and Boehringer Ingelheim, and personal fees from Chugai Pharma, Ono Pharmaceutical, Bristol-Myers, Eli Lilly, MSD, Taiho Pharmaceutical, Pfizer, Novartis and Daiichi Sankyo; outside the submitted work. The other authors have no conflicts of interest to declare.

Ethical Statement: The authors are accountable for all aspects of the work in ensuring that questions related to the accuracy or integrity of any part of the work are appropriately investigated and resolved. The study was conducted in accordance with the Declaration of Helsinki (as revised in 2013). This study was approved by the Sun Yatsen University Cancer Center Institutional Review Board (No. B2020-402-01), and the informed consent (written or verbal) was waived.

Open Access Statement: This is an Open Access article distributed in accordance with the Creative Commons Attribution-NonCommercial-NoDerivs 4.0 International License (CC BY-NC-ND 4.0), which permits the noncommercial replication and distribution of the article with the strict proviso that no changes or edits are made and the original work is properly cited (including links to both the formal publication through the relevant DOI and the license).
See: https://creativecommons.org/licenses/by-nc-nd/4.0/.

\section{References}

1. Siegel RL, Miller KD, Jemal A. Cancer statistics, 2016. CA Cancer J Clin 2016;66:7-30.

2. Borghaei H, Paz-Ares L, Horn L, et al. Nivolumab versus Docetaxel in Advanced Nonsquamous Non-Small-Cell Lung Cancer. N Engl J Med 2015;373:1627-39.

3. Brahmer J, Reckamp KL, Baas $\mathrm{P}$, et al. Nivolumab versus Docetaxel in Advanced Squamous-Cell Non-Small-Cell Lung Cancer. N Engl J Med 2015;373:123-35.

4. Herbst RS, Baas P, Kim DW, et al. Pembrolizumab versus docetaxel for previously treated, PD-L1-positive, advanced non-small-cell lung cancer (KEYNOTE-010): a randomised controlled trial. Lancet 2016;387:1540-50.

5. Rittmeyer A, Barlesi F, Waterkamp D, et al. Atezolizumab versus docetaxel in patients with previously treated non-small-cell lung cancer (OAK): a phase 3, openlabel, multicentre randomised controlled trial. Lancet 2017;389:255-65.

6. Reck M, Rodriguez-Abreu D, Robinson AG, et al. Pembrolizumab versus Chemotherapy for PD-L1Positive Non-Small-Cell Lung Cancer. N Engl J Med 2016;375:1823-33.

7. Herbst RS, Giaccone G, de Marinis F, et al. Atezolizumab for First-Line Treatment of PD-L1-Selected Patients with NSCLC. N Engl J Med 2020;383:1328-39.

8. Sezer A, Kilickap S, Gumus M, et al. Cemiplimab monotherapy for first-line treatment of advanced nonsmall-cell lung cancer with PD-L1 of at least $50 \%$ : a multicentre, open-label, global, phase 3, randomised, controlled trial. Lancet 2021;397:592-604.

9. Ostios-Garcia L, Faig J, Leonardi GC, et al. Safety and Efficacy of PD-1 Inhibitors Among HIV-Positive Patients With Non-Small Cell Lung Cancer. J Thorac Oncol 2018;13:1037-42.

10. Chang E, Sabichi AL, Kramer JR, et al. Nivolumab Treatment for Cancers in the HIV-infected Population. J Immunother 2018;41:379-83.

11. Gay CL, Bosch RJ, Ritz J, et al. Clinical Trial of the Anti-PD-L1 Antibody BMS-936559 in HIV-1 Infected Participants on Suppressive Antiretroviral Therapy. J Infect Dis 2017;215:1725-33.

12. Hentrich M, Schipek-Voigt K, Jager H, et al. Nivolumab in HIV-related non-small-cell lung cancer. Ann Oncol 2017;28:2890.

13. Gonzalez-Cao M, Moran T, Dalmau J, et al. Assessment 
of the Feasibility and Safety of Durvalumab for Treatment of Solid Tumors in Patients With HIV-1 Infection: The Phase 2 DURVAST Study. JAMA Oncol 2020;6:1063-7.

14. Tio M, Rai R, Ezeoke OM, et al. Anti-PD-1/PD$\mathrm{L} 1$ immunotherapy in patients with solid organ transplant, HIV or hepatitis B/C infection. Eur J Cancer 2018;104:137-44.

15. Kothapalli A, Khattak MA. Safety and efficacy of antiPD-1 therapy for metastatic melanoma and non-small-cell lung cancer in patients with viral hepatitis: a case series. Melanoma Res 2018;28:155-8.

16. Lake AC. Hepatitis B reactivation in a long-term nonprogressor due to nivolumab therapy. AIDS 2017;31:2115-8.

17. Pandey A, Ezemenari S, Liaukovich M, et al. A Rare Case of Pembrolizumab-Induced Reactivation of Hepatitis B. Case Rep Oncol Med 2018;2018:5985131.

18. Pertejo-Fernandez A, Ricciuti B, Hammond SP, et al. Safety and efficacy of immune checkpoint inhibitors in patients with non-small cell lung cancer and hepatitis $\mathrm{B}$ or hepatitis C infection. Lung Cancer 2020;145:181-5.

19. Chan GH, Gwee YX, Low JL, et al. Immune checkpoint inhibition for non-small cell lung cancer in patients with pulmonary tuberculosis or Hepatitis B: Experience from a single Asian centre. Lung Cancer 2020;146:145-53.

20. Byeon S, Cho JH, Jung HA, et al. PD-1 inhibitors for non-small cell lung cancer patients with special issues: Real-world evidence. Cancer Med 2020;9:2352-62.

21. Dienstag JL. Hepatitis B virus infection. N Engl J Med 2008;359:1486-500.

22. Liang TJ, Block TM, McMahon BJ, et al. Present and future therapies of hepatitis B: From discovery to cure. Hepatology 2015;62:1893-908.

23. Pardoll DM. The blockade of immune checkpoints in cancer immunotherapy. Nat Rev Cancer 2012;12:252-64.

24. Watanabe T, Bertoletti A, Tanoto TA. PD-1/PD-L1 pathway and T-cell exhaustion in chronic hepatitis virus infection. J Viral Hepat 2010;17:453-8.

25. Cho H, Kang H, Lee HH, et al. Programmed Cell Death 1 (PD-1) and Cytotoxic T Lymphocyte-Associated Antigen 4 (CTLA-4) in Viral Hepatitis. Int J Mol Sci 2017;18:1517.

26. Wang BJ, Bao JJ, Wang JZ, et al. Immunostaining of PD-1/PD-Ls in liver tissues of patients with hepatitis and hepatocellular carcinoma. World J Gastroenterol 2011;17:3322-9.

27. Lin G, Zhuang W, Chen XH, et al. Increase of programmed death ligand 1 in non-small-cell lung cancers with chronic hepatitis B. Ann Oncol 2018;29:516-7.

28. Zhang X, Zhou Y, Chen C, et al. Hepatitis B virus reactivation in cancer patients with positive Hepatitis B surface antigen undergoing PD-1 inhibition. J Immunother Cancer 2019;7:322.

29. Eisenhauer EA, Therasse P, Bogaerts J, et al. New response evaluation criteria in solid tumours: revised RECIST guideline (version 1.1). Eur J Cancer 2009;45:228-47.

30. Terrault NA, Lok ASF, McMahon BJ, et al. Update on prevention, diagnosis, and treatment of chronic hepatitis B: AASLD 2018 hepatitis B guidance. Hepatology 2018;67:1560-99.

31. Fehrenbacher L, Spira A, Ballinger M, et al. Atezolizumab versus docetaxel for patients with previously treated non-small-cell lung cancer (POPLAR): a multicentre, open-label, phase 2 randomised controlled trial. Lancet 2016;387:1837-46.

32. Horn L, Spigel DR, Vokes EE, et al. Nivolumab Versus Docetaxel in Previously Treated Patients With Advanced Non-Small-Cell Lung Cancer: Two-Year Outcomes From Two Randomized, Open-Label, Phase III Trials (CheckMate 017 and CheckMate 057). J Clin Oncol 2017;35:3924-33.

33. Topalian SL, Hodi FS, Brahmer JR, et al. Five-Year Survival and Correlates Among Patients With Advanced Melanoma, Renal Cell Carcinoma, or Non-Small Cell Lung Cancer Treated With Nivolumab. JAMA Oncol 2019;5:1411-20.

34. Gettinger S, Horn L, Jackman D, et al. Five-Year FollowUp of Nivolumab in Previously Treated Advanced NonSmall-Cell Lung Cancer: Results From the CA209-003 Study. J Clin Oncol 2018;36:1675-84.

35. Gandhi L, Rodriguez-Abreu D, Gadgeel S, et al. Pembrolizumab plus Chemotherapy in Metastatic Non-Small-Cell Lung Cancer. N Engl J Med 2018;378:2078-92.

36. Lu S, Cheng Y, Zhou J, et al. Abstract CT218: Flat-dose nivolumab (NIVO) as second-line (2L) treatment (tx) for Asian patients (pts) with advanced non-small cell lung cancer (NSCLC): CheckMate 870. Cancer Res 2020. doi: 10.1158/1538-7445.AM2020-CT218.

37. Koksal AS, Toka B, Eminler AT, et al. HBV-related acute hepatitis due to immune checkpoint inhibitors in a patient with malignant melanoma. Ann Oncol 2017;28:3103-4.

38. Hwang JP, Yilmaz B. Reactivation of Hepatitis B Virus Among Patients With Cancer Receiving Immunotherapy. J Immunother Precis Oncol 2021;4:53-5.

39. He MK, Peng C, Zhao Y, et al. Comparison of HBV 
reactivation between patients with high HBV-DNA and low HBV-DNA loads undergoing PD-1 inhibitor and concurrent antiviral prophylaxis. Cancer Immunol Immunother 2021. [Epub ahead of print]. doi: 10.1007/

$$
\text { s00262-021-02911-w. }
$$

(English Language Editor: J. Gray)

Cite this article as: Zhang $\mathrm{X}$, Tian $\mathrm{D}$, Chen $\mathrm{Y}$, Chen $\mathrm{C}$, He LN, Zhou Y, Li H, Lin Z, Chen T, Wang Y, Russo A, Nadal E, Passiglia F, Soo RA, Watanabe S, Moran T, Oh IJ, Fu S, Hong S, Zhang L. Association of hepatitis B virus infection status with outcomes of non-small cell lung cancer patients undergoing anti-PD-1/PD-L1 therapy. Transl Lung Cancer Res 2021;10(7):3191-3202. doi: 10.21037/tlcr-21-455 